\title{
LES TRANSFERTS DE REPRÉSENTATIONS DANS LES LANGUES (2) : DE PROBLĖME À SUJET
}

\author{
Marie Luce Honeste-SELOSSE \\ Université de Rennes 2
}

\begin{abstract}
This paper deals with the recent transfers of the uses of French word probleme to the word sujet, according to an intralinguistic approach (investigation of the limits of substitutability). The conceptual transfer appears as a result of a cultural transfer, which leads to an alteration of the representations. Nevertheless, the corpus illustrates that speakers are inclined to extend these transfers to set phrases and idiomatic expressions with problème, even if they break the concept carried by sujet.

À Alicja, ma consour en linguistique, avec laquelle une fructueuse et durable collaboration intellectuelle autour de l'amour des mots s'est vite transformée en une fidèle amitié.
\end{abstract}

\section{INTRODUCTION}

Ce travail est la suite d'une étude menée en 2011 sur un phénomène linguistique observé à l'époque en français : l'emploi de souci à la place de problème dans la plupart de ses emplois (Honeste, 2012). Entre temps, dans les cinq années écoulées, on a pu observer un nouveau phénomène, s'ajoutant au précédent : l'utilisation de sujet à la place de problème (et souci). Cette nouvelle étude se situe dans les mêmes perspectives que la précédente : examiner le phénomène du point de vue des conséquences conceptuelles qu'il suppose, dans le cadre théorique que nous avons élaboré (Honeste, 2002, 2006, 2012).

Après un bref rappel de notre méthodologie, nous examinerons dans une première partie le phénomène du point de vue de ses réalisations en français et 
de ses effets sémantiques et conceptuels ; puis, dans une seconde partie, nous chercherons à expliquer l'origine du phénomène et ses conséquences sur le plan conceptuel.

\section{Cadre théorique}

L'étude s'inscrit dans le cadre de la théorie que nous développons depuis de nombreuses années en sémantique lexicale, fondée sur le postulat que toute langue s'inscrit dans une culture donnée et la reflète.

Du point de vue du contenu sémantique des mots, il en découle que, contrairement à la présentation traditionnelle du sens, le signifié des mots n'est pas une description des réalités du monde, mais le résultat des perceptions et des conceptualisations construites dans ce cadre culturel particulier. Le résultat en langue est, à partir de chaque expérience du monde, la formation d'un concept expérientiel ${ }^{1}$, qui constitue le signifié du mot en langue, nécessairement unique et invariant dans tous ses emplois, mais applicable à toute nouvelle expérience rappelant la première. C'est dans le discours que s'observent ces applications, parfois dans des domaines les plus variés, d'où l'interprétation classique de cette variété en termes de "polysémie », confondant signifié de langue et applications de discours.

La méthode suivie sera l'observation d'un corpus d'emplois de sujet à la place de problème $e^{2}$, notamment du point de vue de leur substituabilité, afin de comprendre les raisons et les conséquences de ce changement lexical.

\section{DE PROBLÈME À SUJET}

\subsection{UNE DIFFÉRENCE CONCEPTUELLE...}

De l'étude sémantique de problème, nous avions retenu le substrat étymologique « obstacle, difficulté à résoudre » ${ }^{3}$, qui constitue son signifié de langue.

\footnotetext{
${ }^{1}$ En ce qui concerne la forme et le contenu du concept constituant le signifié de langue des mots, voir Honeste (2006).

${ }^{2}$ Corpus établi entre 2015 et 2018, à partir de discours oraux (captés dans la vie courante, à la radio et la télévision).

${ }^{3}$ Étym. : empr. au lat. problema « question à résoudre » < gr. $\pi \rho o ́ \beta \lambda \eta \mu \alpha ~ «$ ce qu'on a devant soi : obstacle, protection », « ce qui est proposé : question, problème », nom déverbal de $\pi \rho \circ \beta \alpha ́ \lambda \lambda \omega$ (pro « devant » + ballein « jeter ») « jeter devant ; mettre en avant comme argument ; proposer » (Bailly).
} 
Sujet est issu de deux formes latines du verbe subjicere « jeter en dessous $»^{4}$ :

a) du participe passé subjectus, avec la valeur générique "placé dessous, soumis », donnant un adjectif (cf. la forme sougit du droit féodal : " soumis à un maître ») et un nom, avec deux constructions dénotant chacune une forme particulière de sujétion : (i) la construction sujet de, où de introduit la source de sujétion : " (personne, chose) soumise à une autorité »; (ii) la construction sujet à, où à introduit ce vers quoi est tourné le sujet : " (personne, chose) exposée à subir quelque chose (état, action) ». Cette valeur générique issue du latin constitue le concept commun, rémanent (voir Honeste, 2011) dans tous les emplois de ce mot au cours de son histoire ;

b) comme emprunt au latin scolastique subjectum, avec une valeur spécifique (terminologique) abstraite, en philosophie : « ce dont tout le reste s'affirme et qui n'est plus lui-même affirmé d'autre chose » (Aristote, Métaphysique, Z, 3, 1028) en opposition à objectum : « ce qui possède une existence en soi, indépendamment de l'idée que l'on peut en avoir » (Duns Scot) ${ }^{5}$; cette valeur s'étend historiquement à d'autres domaines intellectuels, au XVII ${ }^{\mathrm{e}} \mathrm{s}$. en logique ( « thème »vs « prédicat ») et grammaire (" support d'un propos ») et, plus généralement, dénote " ce qui est soumis à l'observation, la réflexion », appliqué à tout domaine d'étude (sujet de discussion, d'examen, à quel sujet, au sujet de, sans sujet), y compris, par métonymie, à des supports concrets (sujet d'un livre), artistiques (sujet d'un tableau) et humains (sujet de danse, mauvais sujet).

\section{2. ...ENTRAÎNANT DES DIFFÉRENCES DE FONCTIONNEMENT EN DISCOURS}

Pour comprendre pourquoi et quand la substitution en discours est possible ou non entre problème et sujet, il faut préalablement confronter les concepts formant leurs signifiés respectifs, parce que ce sont eux qui déterminent leurs capacités d'emploi en discours. Il nous a paru utile d'intégrer souci à cette confrontation :

- problème : « obstacle, difficulté », i. e. situation imposée de l'extérieur à l'experiencer, nécessitant de sa part une recherche de solution ;

- souci ${ }^{6}$ : « trouble, inquiétude », i. e. état psychologique ressenti par l'experiencer;

- sujet technique: « thème d'observation, d'analyse, de discussion », i. e. posé par l'experiencer.

\footnotetext{
${ }^{4}$ Sauf mention expresse, toutes les analyses de sens et les définitions sont de nous.

${ }^{5}$ Définitions issues de Morfaux (1980).

${ }^{6}$ Étym. : nom déverbal de soucier [v. 1310], du lat. sollicitare « remuer, agiter, inquiéter » (Rey).
} 
Ces exemples d'emploi montrent que les trois mots intègrent une relation à l'experiencer mais selon la gradation suivante : (i) souci dénote une sensation dont la source est interne à l'experiencer ; (ii) problème dénote une situation dont la source est externe, mondaine ; (iii) sujet dénote le résultat d'une action de l'experiencer sur le monde.

Ces différences conceptuelles sont à l'origine des spécificités de fonctionnement des trois mots, notamment du point de vue de leur détermination et de leur combinatoire sémantique en discours :

poser ø / un / des problème(s) - *poser ø / un / des souci(s) vs causer un des souci(s) - *poser sujet (mais un / des possibles en emploi concret métonymique).

La complémentation avec un verbe positionnant le COD comme extérieur à l'experiencer (poser, présenter, etc.) n'est possible qu'avec un COD dénotant lui-même quelque chose d'extérieur à l'experiencer (problème, sujet en emploi métonymique). En revanche, la construction est normalement impossible lorsque le mot dénote quelque chose de lié à l'experiencer, soit de façon interne, comme un sentiment (souci), soit de façon externe, comme le résultat de son action (sujet).

\section{LA SUBSTITUTION DE SUJET À PROBLÈME : EXAMEN DU CORPUS}

Notre corpus des substitutions de sujet à problème présente une différence notable avec celui de souci / problème : la substituabilité de sujet à problème semble être plus aisée et systématique que celle avec souci; de fait, les substitutions sont apparues rapidement et avec une extension plus rapide à tous les emplois de problème. Les occurrences ont été classées à partir des structures d'emploi de problème dans lesquelles elles se sont moulées :

1. «C'est (ce n'est pas) un (mon) problème»

(1) France Inter ${ }^{7}, 05 / 02 / 18$, député : « C'est un sujet qu'il faut porter au débat ».

(2) FI, 07/04/16, journaliste : «L'évolution de la télé ne nous condamne-t-elle pas à des programmes low cost ? »- homme de médias : «C'est un sujet!! C'est un sujet !! ».

\footnotetext{
${ }^{7}$ Désormais FI.
} 
(3) Public Sénat, 28/01/16, scientifique, à propos de "l'empreinte carbone de notre argent" : «L'origine de l'argent, c'était pas un sujet [...], ça devient un sujet maintenant $»$.

(4) FI, 19/01/16, journaliste : « s'il a des problèmes, un déficit d'image, ce n'est pas mon sujet ».

Le présentatif c'est ou ce n'est pas, du fait qu'il introduit une assertion sur la nature de l'objet du discours, est possible avec n'importe quel mot. Cependant, il ne renvoie pas à la même chose selon le concept véhiculé par le mot : l'assertion avec problème renvoie à la difficulté, l'assertion avec sujet renvoie à quelque chose de soumis à la réflexion.

\section{2. «Un vrai (véritable) problème»}

(5) FI, 30/03/15, sociologue : « Il y a un vrai sujet de pouvoir d'achat pour les classes moyennes "».

(6) FI, 29/12/15, journaliste : « Le non recours au RSA est un vrai sujet en France $\gg$.

(7) FI, 25/01/16, analyste politique : " "Est-ce que je suis encore capable de faire ce que je n'ai pas su faire lorsque j'étais au pouvoir", voilà le vrai sujet qui se pose à N. Sarkozy ».

(8) FI, 05/02/18, député : « on a un vrai sujet de la parentalité dans le monde du travail $»$.

(9) FI, 21/08/17, secrétaire d'État à la condition pénitentiaire : « 20000 détenus en attente de jugement, c'est évidemment un sujet»; « des mineurs doublés en cellule, ça, c'est un véritable sujet ».

(10) FI, 10/04/16, présidente de la délégation aux droits des femmes à l'Assemblée nationale : " [à propos du harcèlement sexuel des femmes à l'Assemblée] il faut que les femmes osent porter plainte, c'est là le sujet, et le vrai sujet [...] apporter les preuves, c'est là le vrai sujet ».

Ces occurrences montrent que la caractérisation par vrai ou véritable, ici employés dans leur valeur intensive visant le contenu du concept exprimé (cf. Kleiber, 1994), peut s'appliquer à sujet aussi bien qu'à problème; cependant, l'effet combinatoire n'est pas le même selon le concept véhiculé : appliqué à problème, il intensifie la difficulté, tandis qu'appliqué à sujet, il intensifie la qualité de ce qui est soumis à la réflexion.

\section{3. «Il y a un (petit) problème / Il n'y a aucun problème »}

(11) FI, 11/02/16, le député Thierry Soler, à propos de l'article 49.3 de la Constitution : «Si le Président n'a même pas de majorité au sein de son propre camp, c'est là qu'il y a peut-être un sujet ». 
(12) FI, 13/04/16, invité politique : « Il y a un sujet qui demeure, c'est ... ».

(13) FI, 30/01/18, invité politologue : « Il y a un sujet de pureté » (à propos du foie gras hallal).

(14) FI, 07/04/16, invité sociologue : « Il y a encore un petit sujet, aussi, c'est le temps que les jeunes passent sur Facebook ».

(15) FI, 20/02/17, Directeur de l'Établissement français du sang : « concernant la qualité du sang en France, il n'y a aucun sujet sur le commerce du sang ».

De par son signifié, le présentatif il y a s'applique normalement à quelque chose qui existe en dehors de l'experiencer : la configuration du concept véhiculé par sujet devrait donc en principe bloquer ces emplois (voir infra, « Bilan des emplois de discours $»)$.

\section{4. «Ça pose un problème »}

(16) FI, 06/06/17, invité (à propos de la retraite) : « vivre plus longtemps, avoir trente ans de vie sociale devant soi, ça pose un vrai sujet auquel on n'est pas préparé $\gg$.

(17) FI, 25/01/16, analyste politique : «"Est-ce que je suis encore capable de faire ce que je n'ai pas su faire lorsque j'étais au pouvoir”, voilà le vrai sujet qui se pose à N. Sarkozy ».

Comme expliqué précédemment, le verbe poser ne devrait pas admettre sujet comme COD (voir infra, « Bilan des emplois de discours »).

5. « Avoir un, des problème $(s) »$

(18) FI, 15/10/17, Marwan Muhammad, ancien président du CCIF (Conseil contre l'islamophobie en France) : « concernant l'islamophobie, je n'ai plus de sujet sémantique ; c'est contre le phénomène qu'il faut lutter ».

La rection par avoir, dénotant un lien étroit avec l'experiencer (« détenir, éprouver »), bloque en principe la présence de sujet comme COD (voir infra, « Bilan des emplois de discours »).

6. «Créer des problèmes là où il n'y en a pas », " Prendre un problème à bras-le-corps »

(19) FI, 25/03/16, journaliste : « L'art et la manière de créer des sujets là où il n'y en a pas $\gg$.

(20) FI, 19/05/16, invitée : « [à propos du co-voiturage] il y a des mutuelles qui prennent le sujet à bras-le-corps ». 
Ces deux expressions lexicalisées se trouvent ici modifiées par le remplacement de problème par sujet, et ce, dans une situation où l'action porte sur un objet totalement extérieur à l'experiencer, en cohérence avec la conceptualisation que véhicule problème, mais pas avec celle véhiculée par sujet.

Le classement du corpus présenté ci-dessus met en évidence la substitution de sujet à problème dans la plupart des emplois de ce dernier, y compris dans ses emplois figés, et même jusque dans des expressions lexicalisées, comme le montrent les deux dernières occurrences.

\section{Bilan DES OBSERVATIONS}

\subsection{EFFETS CONCEPTUELS DES SUBSTITUTIONS}

On constate que dans toutes les occurrences du corpus de substitutions par sujet, problème renvoie à l'idée d'obstacle, de difficulté. Il en était de même dans le corpus de substitutions par souci. On en déduit donc que la substituabilité des trois mots est nécessairement restreinte aux situations d'emploi renvoyant à un obstacle, une difficulté. De ce fait, la substitution doit s'interpréter comme une volonté des locuteurs de mettre en place une conceptualisation différente d'une même expérience : celle de la difficulté. Nous avons expliqué supra les différences conceptuelles véhiculées par ces trois mots, qu'il s'agit donc à présent de rapporter au contexte commun de difficulté :

- problème dénote une situation qui constitue une difficulté pour l'experiencer ;

- son substitut souci dénote l'état de trouble de l'experiencer d'une difficulté ;

- son substitut sujet dénote la soumission d'une difficulté à la réflexion de l'experiencer.

Notre étude précédente avait montré que le transfert conceptuel opéré de problème à souci devait s'interpréter comme une volonté de ramener la difficulté à la seule sensation éprouvée par l'experiencer. Sujet, pour sa part, a pour rôle de ramener la difficulté à une simple matière soumise à l'observation de l'homme. Cette nouvelle substitution s'inscrit donc dans un même mouvement d'engagement de plus en plus important de l'experiencer dans son expérience, lui prêtant un rôle de plus en plus actif dans son rapport à la difficulté. 


\section{DES ChANGEMENTS CONCEPTUELS AUX CALQUES D'EMPLOIS}

Comme dans le corpus des remplacements de problème par souci, on observe que les remplacements de problème par sujet tendent à s'étendre à tous les emplois de problème conceptuellement compatibles, notamment les expressions figées ; plus précisément, ce qui se produit est que sujet remplace souci dans les emplois de problème!

En revanche, sujet apparaît dès les premières observations dans des contextes où la substitution n'est pas possible sans créer de conflit de conceptualisation, situation qui ne s'était pas présentée avec souci au moment de la constitution du premier corpus ; cependant, nous avons aussi pu relever récemment quelques situations d'emploi problématiques pour souci. Nous allons donc revenir sur ces situations d'emploi " illégitimes », désormais présentes dans nos deux corpus, pour tenter d'en donner une explication.

Les occurrences problématiques du corpus problème / souci sont les suivantes :

(21) FI, 25/11/17, le dessinateur Johann Sfarr [présentant son $7^{\mathrm{e}}$ opus du Chat du Rabin] : « [il le fait], même si ça lui attire des soucis ».

(22) Bulletin communal Cusy infos $\mathrm{n}^{\circ} 22$ - décembre 2017, p. 7 : « La présence de chats "libres" sur la commune pose des soucis (odeurs, dégâts dans les poubelles...)».

Dans la première occurrence, la collocation attirer des soucis apparaît comme une collision de deux expressions figées : causer des soucis et attirer des ennuis. Dans la seconde, le même phénomène se produit entre poser des problèmes et causer des soucis. Ces deux occurrences participent donc d'un même principe d'attraction d'expressions figées présentant une formation identique (v. inf. + COD n. plur.) et dénotant toutes un impact sur l'experiencer; la collision est encore facilitée par la proximité phonique dans le cas du passage de causer à poser, à partir de l'expression causer des soucis.

Dans le corpus problème / sujet, on a relevé un nombre plus important d'occurrences problématiques, construites soit avec le présentatif ily a (occurrences 11 à 15), soit avec des verbes comme avoir (occurrence 18) et poser (occurrences 16 et 17), ces dernières étant à rapprocher de celles du corpus précédent.

Ce phénomène observé dans les deux corpus peut être considéré comme un effet de systématisation des substitutions chez les locuteurs, accoutumés à parler de souci ou de sujet plutôt que de problème, qui les conduit à pratiquer spontanément le calque d'emploi dans toutes les occurrences de discours. Le procédé du calque d'emploi apparaît de façon évidente en classant les occurrences d'un mot sous 
les structures d'emploi de l'autre, comme nous l'avons fait supra pour sujet. L'aboutissement de ce mécanisme de substitution systématique est la présence du mot-substitut y compris dans des expressions lexicalisées, comme l'illustrent les deux dernières occurrences du corpus de sujet.

On comprend alors que la logique de substitution finisse par dépasser les contraintes conceptuelles au profit de la systématicité, en admettant des emplois que le sémanticien reste alors seul à percevoir comme abusifs...

\section{Conclusion}

Les trois mots problème, souci et sujet ont en commun de dénoter des expériences de l'homme et non des objets du monde.

Notre étude précédente sur problème et souci avait montré que ces deux mots ont en commun de parler de l'expérience humaine d'une difficulté, ce qui explique leur substituabilité, tout en véhiculant des conceptualisations différentes de cette expérience : problème dénote quelque chose qui constitue un obstacle pour l'homme; souci parle du sentiment de trouble que l'homme éprouve. De ce fait, le remplacement de problème par souci doit s'interpréter comme une volonté d'opérer un transfert conceptuel, visant à recentrer l'expérience de la difficulté sur l'experiencer.

Sujet se démarque des deux mots précédents en ce qu'il dénote ce que l'homme soumet à son observation (cf. son étymologie). Donc sujet, comme les deux précédents, intègre dans son signifié le rapport de l'homme au monde, mais cette fois-ci comme acteur (agent dans le schéma actanciel du déverbal étymologique).

Il y a donc une intéressante évolution du rapport de l'homme au monde dans cette succession rapide des substitutions lexicales :

- problème renvoie à l'effet de l'objet sur l'homme, où est pris en compte le phénomène mondain en cause, dans la mesure où il implique une action de résolution;

- souci renvoie à la seule sensation éprouvée, le phénomène du monde n'étant même pas présenté comme objet de manipulation ;

- sujet dénote l'objet d'une manipulation (sujétion) de l'homme.

On avait déduit du passage de problème à souci, un recentrage de l'expérience de la difficulté sur l'experiencer ; on peut de même déduire du passage de souci à sujet, une promotion de l'experiencer passif à un rôle d'agent, en même temps qu'une oblitération de la notion de difficulté. 
On peut alors se demander quelle est la raison de cette succession logique des transferts conceptuels. On peut peut-être y trouver une réponse dans l'examen de l'origine de ces substitutions. Nos corpus comportent essentiellement des occurrences tirées des médias, portant essentiellement la parole de gens de la politique et des médias ; si notre corpus est ainsi fait, c'est que ces substitutions, dans leurs premières occurrences, n'avaient jamais pour source la conversation courante. On peut donc s'autoriser à chercher des éléments de réponse à partir de l'analyse des caractéristiques sociales des locuteurs.

Cette population a pour particularité commune d'être dans la proximité des pouvoirs ; on peut alors bien imaginer que, dans cette classe particulière qui a intérêt à valoriser autant que possible ses agissements, les problèmes soient quelque chose qu'on ne tient pas à souligner. Après les avoir déplacés de la sphère collective vers les individus avec souci, on peut penser que l'étape suivante consiste à les atténuer, en supprimant l'élément néfaste : l'obstacle, la difficulté. Sujet est alors un excellent candidat à la substitution, puisqu'il n'offre plus qu'une matière à discuter dépouillée de tout aspect fâcheux... voire fâchant.

\section{Références bibliographiques}

\section{Ouvrages et articles}

ARISTOTE (1991), La Métaphysique (trad. et commentaires par J. Tricot), Paris, Librairie Philosophique J. Vrin.

HONESTE, Marie Luce (2002), «Organisation du lexique et identité culturelle », in Translation and Meaning Part 6. Proceedings of the Lódź Session of the 3rd International Maastricht - Łódź Duo Colloquium, Łódź, Poland, 22-24/09/2000 (B. Lewandowska-Tomaszczyk, M. Thelen éds), Maastricht, Universitaire Pers Maastricht, pp. 211-221.

HONESTE, Marie Luce (2006), «Approche cognitive du sens lexical », in Actes du XXIV Congrès International de Linguistique et de Philologie Romanes (D. A. Trotter éd.), vol. IV, Tübingen, Niemeyer Verlag, pp. 105-118.

HONESTE, Marie Luce (2011), « Le phénomène de rémanence et ses conséquences en sémantique lexicale à travers l'histoire du mot opinion », Le Français préclassique, n 13 , Paris, Honoré Champion, p. 91-113.

HONESTE, Marie Luce (2012), « Les transferts de représentations dans les langues : de worry à souci et de problème à souci, un exemple de calque culturel en français de France ", in Pluralité des cultures : chances ou menaces. Analyses linguistiques et didactiques (A. Kacprzak,

A. Konowska, M. Gajos éds), Łódź-Lask, Oficyna Wydawnicza LEKSEM, pp.155-164.

KLEIBER, Georges (1994), Anaphores et pronoms, Louvain-la-Neuve, Duculot.

MORFAUX, Louis-Marie (1980), Vocabulaire de la philosophie et des sciences humaines, Paris, Armand Colin. 


\section{Dictionnaires}

BAILLY, Antoine (1950), Dictionnaire grec-français, Paris, Hachette.

GAFFIOT, Félix (1934), Dictionnaire latin-français, Paris, Hachette.

GREIMAS, Algirdas J. (1994), Dictionnaire de l'ancien français, Paris, Larousse.

GUILBERT, Louis, LAGANE, René, NIOBEY, Georges (1986), Grand Larousse de la Langue Française, 7 vol., $2^{\mathrm{e}}$ éd., Paris, Larousse.

IMBS, Paul, QUEMADA, Bernard (dir.) (1971-1994), Trésor de la langue française. Dictionnaire de la langue du XIXe et du XXe siècle (1789-1960), 16 vol., Nancy, CNRS-INAL / Paris, Gallimard. REY, Alain (dir.) (1992), Dictionnaire historique de la langue française, Paris, Dictionnaires Le Robert. 